\title{
Pharmacist-led screening in sexually transmitted infections: current perspectives
}

This article was published in the following Dove Press journal: Integrated Pharmacy Research and Practice

\section{Helen Wood \\ Sajni Gudka}

School of Allied Health, Faculty of Health and Medical Sciences, The University of Western Australia, Perth, WA, Australia
Correspondence: Sajni Gudka School of Allied Health, Faculty of Health and Medical Sciences, University of Western Australia, M315, Curnow Building, Nedlands, Perth, WA 6009, Australia

Tel +61864887510

Fax +6I 864887532

Email sajni.gudka@uwa.edu.au
Introduction: Sexually transmitted infection (STI) screening is a crucial initiative that aims to reduce the increasing global prevalence of many common STIs such as chlamydia, gonorrhea, and herpes simplex virus (HSV). Many STIs are either asymptomatic or show mild symptoms that are often attributed to other infections; hence, screening is the only way to identify - and by extension, treat - them. In this way, the spread of STIs can be reduced, and the health implications of an untreated STI are minimized. Community pharmacies could provide an avenue to convenient, confidential STI screening by using noninvasive or minimally invasive sample collection techniques that are used by the consumer or pharmacist. We identified the most common STIs found globally and investigated the current and potential role of pharmacists in provision of STI screening interventions.

Discussion: There is sufficient evidence for pharmacy-based chlamydia screening, with many consumers and pharmacists finding it an acceptable and highly valued service. Some evidence was found for pharmacy-based gonorrhea, hepatitis B virus (HBV), and human immunodeficiency virus (HIV) screening. Appropriate sample collection for gonorrhea screening needs to be further examined in a pharmacy setting. HBV screening presented an increased risk of personal injury to pharmacists through the collection of whole blood specimens, which could be reduced through consumer self-sampling. Pharmacist-collected specimens for HIV is less risky as an oral swab can be used, nullifying the risk of transmission; but pre- and post-screen consultations can be time-intensive; hence, pharmacists would require remuneration to provide an ongoing HIV screening service. Not enough evidence was found for syphilis screening through community pharmacies; more studies are required that consider sampling methods other than pharmacist-collected whole blood specimens. There is no evidence to date for pharmacist-led trichomoniasis or HSV screening.

Conclusion: Pharmacists are well-positioned to provide STI screening services, but further investigations are needed to overcome financial, safety, and confidentiality barriers.

Keywords: pharmacist, screening, sexually transmitted infection, testing, chlamydia, gonorrhea

\section{Introduction}

The prevalence of sexually transmitted infections (STIs) is a global health concern, with more than 1 million curable STIs acquired worldwide every day. ${ }^{1}$ There are currently $>30$ microorganisms including bacteria, viruses, and parasites that are transmissible through vaginal, anal, or oral sex or genital skin to skin contact. ${ }^{1,2}$ In addition, some STIs can be transmitted through blood or blood products, or passed from mother to child during pregnancy, childbirth, or through breastmilk. ${ }^{1,3}$

submit your manuscript $\mid$ www.dovepress.com


Although most bacterial STIs are frequently asymptomatic, common symptoms include vaginal or rectal discharge, urethral discharge or burning in men, abdominal pain, or genital ulceration. ${ }^{4}$ Early symptoms of viral STIs include small fluid-filled blisters or flesh-colored bumps around the genitals, anus, or oral cavity, systemic flu-like symptoms, or abdominal pain; while protozoal STIs could cause itching due to the presence of egg sacs or lice in pubic hair, unpleasant vaginal discharge or pain during intercourse.

There are eight pathogens that contribute to a significant portion of the worldwide prevalence of STIs. Four of these - chlamydia, gonorrhea, syphilis, and trichomoniasis - are curable. Four viral infections - human papillomavirus (HPV), hepatitis B virus (HBV), herpes simplex virus (HSV), and human immunodeficiency virus (HIV) - are incurable, but the symptoms can be managed with treatment. The symptoms and sequelae of these eight STIs are detailed in Table 1.

STIs are often more prevalent in developing countries where easy access to asymptomatic "screening" and symptomatic "testing" are unavailable, prohibitively expensive, or inaccessible. Particularly, the impact of incurable viral STIs such as HIV has been devastating to residents' health and well-being. ${ }^{1,13}$ Furthermore, the World Health Organization (WHO) estimates that up to $90 \%$ of all curable bacterial and parasitic infections are found in the residents of developing countries. ${ }^{1}$ Detection of STIs in this setting often relies on diagnosis by symptomatic identification; as such, many asymptomatic infections go undetected and therefore untreated. ${ }^{1}$ Conversely, coordinated and well-funded health services in developed countries generally provide easy access to accurate diagnostic testing, which helps for prompt diagnosis and treatment of asymptomatic infections. ${ }^{1}$

A basic but well-accepted epidemiology formula could be used to explain the three parameters that affect the rate of spread of STIs $\left(\mathrm{R}_{0}\right)$ in a population, ${ }^{14}$ namely probability of transmission, contact rate, and duration of infection. The relationship between these parameters can be illustrated by the following formula:

$$
\mathrm{R}_{0}=\beta \times \mathrm{k} \times \mathrm{D}
$$

Table I Symptoms and sequelae of the most significant STIs

\begin{tabular}{|c|c|c|c|c|}
\hline \multirow{2}{*}{$\begin{array}{l}\text { Infection } \\
\text { Causative organism } \\
\text { Chlamydia }^{5}\end{array}$} & \multicolumn{2}{|c|}{ Symptoms } & \multicolumn{2}{|c|}{ Possible sequelae } \\
\hline & $0^{\pi}$ & Genital or rectal pain discharge & $0^{\pi}$ & Reactive arthritis \\
\hline Chlamydia trachomatis & $\sigma^{*}$ & Burning sensation on urination & $q^{x}$ & Infertility or reduced fertility \\
\hline \multirow[t]{3}{*}{ bacterium } & q & Painful sexual intercourse & $0^{7}$ & Epididymo-orchitis \\
\hline & \% & Lower abdominal pain & \% & Pelvic inflammatory disease \\
\hline & & & q & Ectopic pregnancy \\
\hline Gonorrhea $^{6}$ & $q^{\pi}$ & Genital or rectal pain, discharge & $q^{n}$ & Disseminated infection, affecting joints, skin, heart \\
\hline Neisseria gonorrhoeae & $q^{n}$ & Burning sensation on urination & $q^{n}$ & Infertility or reduced fertility \\
\hline \multirow[t]{2}{*}{ bacterium } & q & Painful sexual intercourse & $\sigma^{\prime}$ & Epididymo-orchitis \\
\hline & & & ९ & Pelvic inflammatory disease \\
\hline Syphilis ${ }^{7}$ & $q^{n}$ & Oral, anal, or genital ulceration & $q^{x}$ & Skin lesions \\
\hline Treponema pallidum & $q^{\pi}$ & Generalized rash & $q^{n}$ & Cardiovascular, neurological disease \\
\hline bacterium & $q^{*}$ & Fever, malaise & & \\
\hline \multirow[t]{2}{*}{ Trichomoniasis $^{8}$} & $q^{\pi}$ & Genital discharge & $0^{x}$ & Prostatitis \\
\hline & $\sigma^{3}$ & Burning sensation on urination & q & Risk of preterm delivery in pregnant women \\
\hline Trichomonas vaginalis & q & Vulval itching & q & Risk of postpartum sepsis \\
\hline protozoan & \% & Cervicitis & & \\
\hline \multirow[t]{4}{*}{ Herpes simplex virus ${ }^{9}$} & $q^{x}$ & Itchy, painful genital or anal blisters or ulcers & $q^{\pi}$ & Urinary bladder malfunction \\
\hline & $q^{\pi}$ & Genital or rectal itching & $q^{\pi}$ & Psychosexual morbidity \\
\hline & $0^{\pi}$ & Urethritis or proctitis & q & Transmission to fetus \\
\hline & 우 & Cervicitis & & \\
\hline \multirow[t]{3}{*}{ Hepatitis $B$ virus ${ }^{10}$} & $q^{\pi}$ & Flu-like symptoms and general malaise & $q^{\pi}$ & Cirrhosis \\
\hline & $q^{\pi}$ & Jaundice & $q^{\pi}$ & Hepatocellular carcinoma \\
\hline & $q^{\pi}$ & Pale stools and dark urine & & \\
\hline \multirow[t]{3}{*}{ Human papillomavirus" } & $q^{\pi}$ & Genital or anal warts, usually painless & $0^{\prime}$ & Penile, anal, oropharyngeal malignancy \\
\hline & $q^{\pi}$ & Perianal itching & q & Vulvar, vaginal, cervical, anal, oropharyngeal malignancy \\
\hline & $q^{\pi}$ & Perirectal bleeding & & \\
\hline \multirow{3}{*}{$\begin{array}{l}\text { Human immunodeficiency } \\
\text { virus }(\mathrm{HIV})^{12}\end{array}$} & $q^{\pi}$ & Flu-like symptoms & $q^{\top}$ & Opportunistic infections such as pneumonia, esophageal \\
\hline & $q^{\pi}$ & Generalized rash & & candidiasis \\
\hline & & & $q^{\pi}$ & Kaposi's sarcoma \\
\hline
\end{tabular}

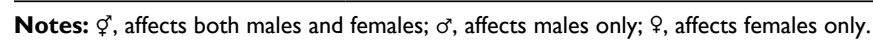


$R_{0}$ is the rate of spread of STI in the population; $\beta$ is the probability of transmission: how easily the pathogen can be transferred from an infected to a susceptible individual; $\mathrm{k}$ is the contact rate: the rate of contact between infected and susceptible individuals; D is the duration of infection: how long the infection persists in the individual.

If $\mathrm{R}_{0}<1$, the infection will eventually disappear, if $\mathrm{R}_{0}=1$, the infection will become endemic (disease found regularly in the population), and if $\mathrm{R}_{0}>1$, there will be an epidemic (disease very common in the population). Even though there are some objections to the generality of this formula, it has been mathematically proven that these three factors equally influence the rate in which STIs spread in society. ${ }^{14}$ A 20\% decrease in one of these factors will have the same effect as a $20 \%$ decrease in any of these factors.

Promoting safe sex and condom use could reduce $\beta$, while promoting educational interventions - resulting in fewer sexual partners and concurrent partnerships - could reduce $\mathrm{k}$. However, the particular challenge is reducing $\mathrm{D}$ - the mean duration of infection. Since many STI-positive individuals are asymptomatic, they have no reason to seek health care. Unless they present for a check-up or are notified by a partner who has developed symptoms, they may remain infectious for a long period. This is where the health care system can directly intervene by implementing STI screening interventions that target potentially infected people from an apparently asymptomatic population.

STI screening requirements are determined by an individual's sexual history, personal risk factors for infection, and local clinical guidelines. ${ }^{15,16}$ It involves collecting at least one biological sample (urine, blood, cells, or fluid) for analysis and diagnosis. ${ }^{15}$ If necessary, treatment is guided by the results, and STI-positive people are encouraged to notify their recent sexual partners of their potential exposure.

Screening interventions can identify many recently acquired or persistent STIs. When STI status is known, treatment or management strategies can be accessed to avoid or reduce long-term complications and lower the chances of the infection spreading to others. ${ }^{17}$ In addition, recent sexual partners can be notified to prompt them to seek STI screening, when they otherwise may not have. ${ }^{17}$ When utilized, the benefits of STI screening interventions can be seen on an individual, national, and global scale. Individuals who are diagnosed - and by extension, treated - because of screening can receive the health and/or financial benefits associated with early intervention. ${ }^{18}$ National benefits can be attributed to reduced morbidity and mortality associated with STIs, and there are resultant economic benefits such as reduced expenditure for health services or reduced loss of productivity. ${ }^{18}$ As STIs are a global epidemic, coordinated national screening programs can result in fewer STI-positive individuals traveling internationally and propagating the spread of infection. ${ }^{18,19}$

Community pharmacies could be an appropriate setting for some STI screening. ${ }^{20}$ Their convenient location and opening hours improve accessibility, and pharmacists are seen as trusted health professionals in a highly regulated industry. ${ }^{21}$ In addition, pharmacists can offer private consultations in a health care setting that is already involved in sexual health through sales of condoms and the provision of emergency hormonal contraceptive (EHC) and oral contraceptives. ${ }^{20,22}$

In this review, we have collated the current and potential role of community pharmacists in screening interventions for STIs.

\section{Methodology}

Three databases (EMBASE, Medline, and Global Health) were searched for relevant articles reporting on original research up to August 31, 2017. The following search terms were used:

- "Sexually transmi*" OR chlamydia OR gonorrhea OR gonorrhoea OR syphilis OR “Hepatitis B” OR HIV OR "Human Immunodeficiency Virus" OR trichomoniasis OR HSV OR "Herpes Simplex Virus"; AND

- Pharmac*; AND

- Testing OR screening.

Following full-text screening, 15 relevant articles were selected. A further three articles were included from the authors' own reference libraries.

\section{Chlamydia}

\section{Background}

WHO estimates that every year there are around 131 million new chlamydial infections globally; latest global estimates of annual new infections by region are shown in Figure $1 .{ }^{1}$ Recent data indicate that females have a higher incidence rate over males in every global geographic region for a total of $4.2 \%$ versus $2.7 \%$ positivity rates, respectively. ${ }^{23}$ Those at greatest risk of infection are sexually active people aged 15-29 years, individuals with two or more sexual partners in the preceding year, individuals with a new sexual partner, and men who have sex with men. ${ }^{24,25}$ 


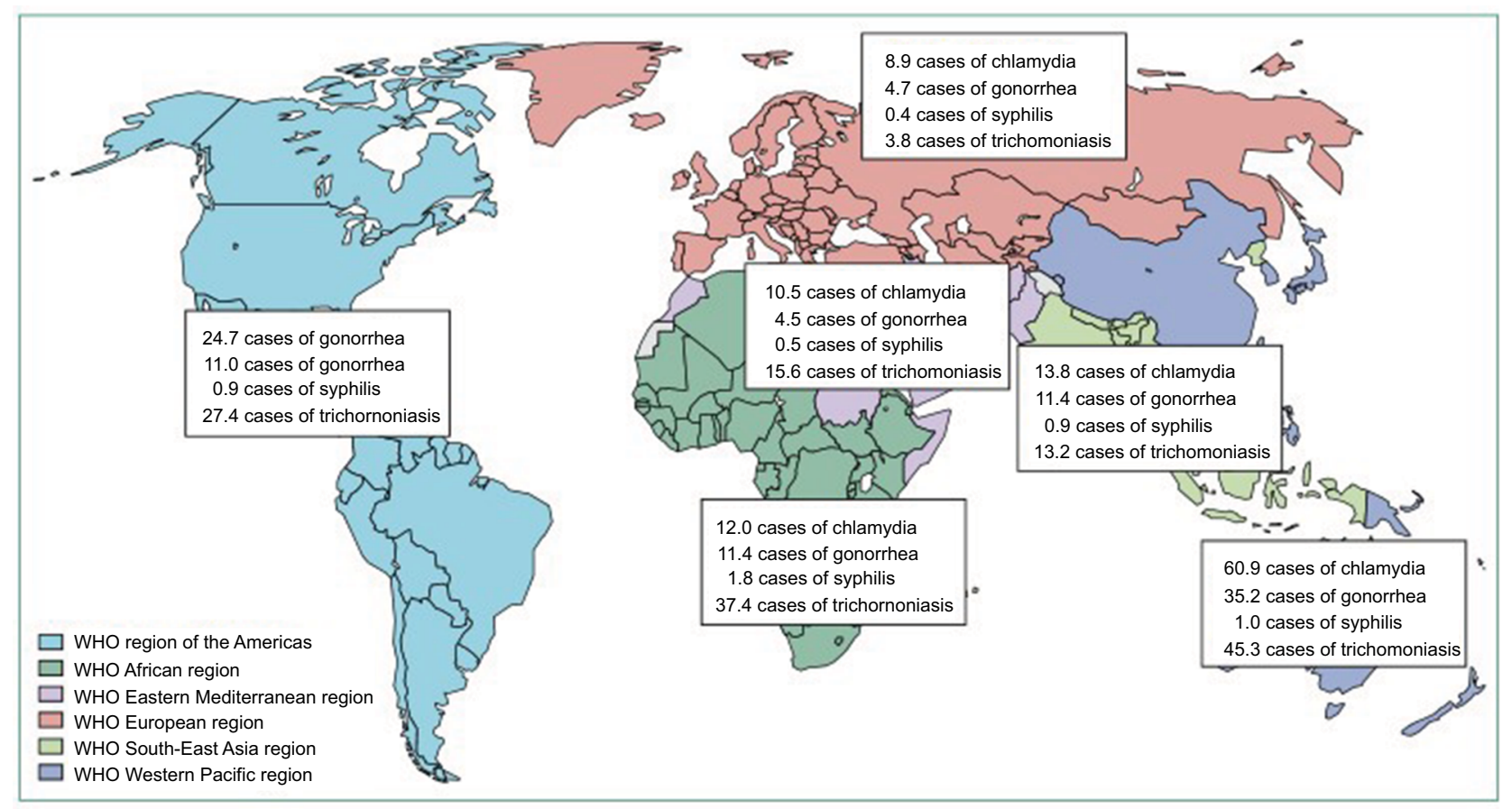

Figure I 2012 WHO estimate of number in millions of new cases of the four curable STIs, by world region.

Note: Reprinted from Lancet Infect Dis, 17(8), Unemo M, Bradshaw CS, Hocking JS, et al, Sexually transmitted infections: challenges ahead, e235-e279, Copyright (2017), with permission from Elsevier. ${ }^{16}$

Abbreviations: WHO, World Health Organization; STI, sexually transmitted infection.

Control of chlamydia is extremely challenging because $70 \%-90 \%$ of infected women and over $50 \%$ of infected men are asymptomatic and can remain this way for years. ${ }^{26,27}$ If the infection is not detected and treated, chlamydia-positive individuals would spread the infection to subsequent sexual partners - even in the absence of symptoms. Left untreated, persistent chlamydia lead to serious and potentially permanent sequelae such as pelvic inflammatory disease and tubal factor infertility in women, or epididymitis and epididymoorchitis in men. ${ }^{27}$ Screening is therefore essential to detect chlamydia in asymptomatic individuals to prevent the spread of infection and minimize the potential for complications arising from untreated chlamydia.

\section{Current practice}

Screening for chlamydia typically involves testing a specimen by using Nucleic Acid Amplification Testing (NAAT). ${ }^{5,28,29}$ NAAT is the preferred test recommended by WHO as it is highly sensitive and specific and can be used for a wide range of samples..$^{29}$ The type of specimen collection varies depending on gender and risk factors (Table 2). To access chlamydia screening from a traditional setting, the consumers usually needs to make and attend an appointment with their general practitioner (GP), or present to a family planning clinic or sexual health clinic. The health provider or consumer collects the relevant specimens as shown in Table 2 for screening and waits for the pathology result before commencing treatment if positive.

Once diagnosed, chlamydia is easily treated with readily available antibiotics. The management guidelines in the USA, England, and Australia recommend the use of azithromycin (1 g orally, single dose) or doxycycline (100 mg orally, twice daily for 7 days) as first-line treatment for uncomplicated chlamydial infections. ${ }^{5,31,32}$

Well-documented barriers to the existing screening pathways include embarrassment, inconvenience, fear of invasive sample collection, and low motivation to self-refer for screening. ${ }^{33-36}$ Consequently, screening rates in general practice remain low; and therefore, alternative sources of chlamydia screening may be warranted. ${ }^{37,38}$ Community pharmacists have been identified as a possible provider of chlamydia screening because the convenience and accessibility offered by pharmacies overcome some of the common barriers faced in chlamydia screening in a traditional setting. ${ }^{39}$

\section{Role of the pharmacist}

Currently, chlamydia screening kits can be purchased from some pharmacies in the USA and the UK, with some UK 
pharmacies also providing free screening kits over the counter. ${ }^{40-43}$ Chlamydia screening kits come with everything needed to self-collect a urine or vaginal swab sample and a prepaid postage bag to return the sample for analysis and diagnosis..$^{40}$ Results are relayed to the consumer via telephone, text messaging, email, or a website with a secure log-in, and the consumer is directed to an appropriate service for treatment if necessary. ${ }^{40}$

To date, the National Chlamydia Screening Programme in the UK is the only coordinated screening initiative that involves community pharmacists. ${ }^{44}$ UK pharmacists are able to provide asymptomatic consumers with a chlamydia screening kit. ${ }^{44}$ The consumers collect their own urine or vaginal swab samples and post them to a designated testing facility. ${ }^{44}$ If a consumer is chlamydia-positive, the pharmacist can arrange treatment and discuss partner notification - another crucial component of chlamydia management. ${ }^{44}$

Gudka previously identified five core requirements that should be considered when designing future pharmacy-based chlamydia screening interventions. ${ }^{45}$ We have reviewed the evidence for the uptake, acceptability, and barriers of key STI screening initiatives conducted from community pharmacies with these five core requirements in mind (Table 4).
In summary, our literature review shows that pharmacistled chlamydia screening studies have been conducted in the USA, the Netherlands, England, and Australia (Table 4). The timescale of interventions ranged from 2 weeks to 24 months, and the numbers of completed screenings spanned from 18 to 900. Despite the broad range, the key finding is that the concept of pharmacy-accessed chlamydia screening is feasible and acceptable to pharmacists and consumers. Consumers highly valued the convenience of self-collected samples, particularly when they could immediately collect the sample and hand it to pharmacy staff, as opposed to returning samples by post or delivering to pathology drop-off points. Pharmacists were keen to continue providing chlamydia screening beyond the scope of the research and found that opportunistically offering screening to at-risk consumers did not considerably increase their workload or time spent per consultation - particularly when discussed during a sexual health consultation.

\section{Summary}

There is sufficient evidence that community pharmacists can have a role in community-based chlamydia screening. The core requirements identified by Gudka et al could be used in developing future interventions, because they set a framework

Table 2 Specimens collected for chlamydia screening

\begin{tabular}{ll}
\hline Specimen & When collected \\
\hline First void urine & ơ All \\
& o Only if endocervical swab or vaginal swab cannot be taken, but not as sensitive as vaginal swab \\
Anorectal swab & ơ All men who have sex with men \\
& \% If patient has had anal sex \\
Pharyngeal swab & ơ All men who have sex with men \\
& ᄋ If patient has had oral sex \\
Endocervical swab & क Collected upon examination \\
Vaginal swab & क Collected when no examination
\end{tabular}

Notes: $\sigma^{\prime}$, males; 9 , females; ${ }^{a}$ cannot be self-collected. Data from Australasian Sexual Health Alliance ${ }^{5}$ and Government of Western Australia Department of Health. ${ }^{30}$

Table 3 Core requirements for consideration when designing a pharmacy-based chlamydia screening intervention

\section{Core requirement}

Consumer recruitment approach

Consumer eligibility criteria

Specimen testing/handling procedure

Arrangement for notifying test results Management of chlamydia-positive patients and partners

Note: Data from Gudka. ${ }^{45}$
Population-based screening - anyone can obtain a chlamydia test from a pharmacist

Opportunistic screening - pharmacists approach consumers who fit certain criteria, eg, women purchasing emergency hormonal contraception

Restricted by age

Restricted by gender

Specimen - noninvasive such as first void urine or vaginal swab sample

Collected samples - posted directly to pathology laboratory or consumer-delivered to a designated collection point

Usually done by a dedicated chlamydia screening officer

Antibiotic therapy can be obtained from general practitioner, sexual health clinic, or community pharmacist (following specific protocol if no prescription) 
Table 4 Summary of characteristics, core requirements, and results of pharmacist-led sexually transmitted infection screening studies

\begin{tabular}{|c|c|c|c|c|c|c|c|}
\hline \multicolumn{8}{|c|}{ Chlamydia screening studies } \\
\hline \multicolumn{2}{|l|}{ Quantitative } & \multicolumn{5}{|c|}{ Core requirements } & \multirow[b]{2}{*}{ Uptake } \\
\hline $\begin{array}{l}\text { Author, year } \\
\text { country }\end{array}$ & Time frame & $\begin{array}{l}\text { Recruitment } \\
\text { approach }\end{array}$ & $\begin{array}{l}\text { Consumer } \\
\text { eligibility }\end{array}$ & $\begin{array}{l}\text { Type of } \\
\text { specimen; } \\
\text { method } \\
\text { of sample } \\
\text { return }\end{array}$ & $\begin{array}{l}\text { Result } \\
\text { notification }\end{array}$ & $\begin{array}{l}\text { Treating } \\
\text { health } \\
\text { professional }\end{array}$ & \\
\hline $\begin{array}{l}\text { Bloomfield } \\
\text { et al, } 2002^{46} \\
\text { USA }\end{array}$ & 2 weeks & Population & NR & $\begin{array}{l}\text { FVU; } \\
\text { Postal return }\end{array}$ & Telephone & $\begin{array}{l}\text { Pharmacist, GP } \\
\text { or sexual health } \\
\text { clinician }\end{array}$ & $\begin{array}{l}\text { Distributed: } 209 \\
\text { Returned: } 80 \\
(38 \%)\end{array}$ \\
\hline $\begin{array}{l}\text { van Bergen } \\
\text { et al, } 2004^{47} \\
\text { The }\end{array}$ & 24 months & $\begin{array}{l}\text { Opportunistic } \\
\text { (contraceptive } \\
\text { prescription) }\end{array}$ & $\begin{array}{l}\text { Female } \\
\text { I5-29 years }\end{array}$ & $\begin{array}{l}\text { FVU; } \\
\text { Postal return }\end{array}$ & Telephone & $\begin{array}{l}\text { GP or sexual } \\
\text { health clinician }\end{array}$ & $\begin{array}{l}\text { Eligible: } 614 \\
\text { Offered: } 446 \\
(73 \%)\end{array}$ \\
\hline Netherlands & & & & & & & $\begin{array}{l}\text { Returned: } 121 \\
(27 \%)\end{array}$ \\
\hline $\begin{array}{l}\text { Baraitser et al, } \\
2007^{48} \\
\text { England }\end{array}$ & 3 months & $\begin{array}{l}\text { Population and } \\
\text { opportunistic } \\
\text { (EHC } \\
\text { consultation) }\end{array}$ & NR & NR & NR & $\begin{array}{l}\text { Pharmacist, GP, } \\
\text { or sexual health } \\
\text { clinician }\end{array}$ & Returned: 83 \\
\hline $\begin{array}{l}\text { Gudka et al, } \\
2009^{49} \\
\text { Australia }\end{array}$ & 6 months & $\begin{array}{l}\text { Opportunistic } \\
\text { (EHC } \\
\text { consultation) }\end{array}$ & $\begin{array}{l}\text { Female } \\
\geq 18 \text { years }\end{array}$ & $\begin{array}{l}\text { Vaginal swab; } \\
\text { Pathology } \\
\text { laboratory } \\
\text { or issuing } \\
\text { pharmacy }\end{array}$ & Telephone & $\begin{array}{l}\text { GP or sexual } \\
\text { health clinician }\end{array}$ & $\begin{array}{l}\text { Distributed: } 166 \\
\text { Returned: } 46 \\
(28 \%)\end{array}$ \\
\hline $\begin{array}{l}\text { Brabin et al, } \\
2009^{22} \\
\text { England }\end{array}$ & 12 months & $\begin{array}{l}\text { Opportunistic } \\
\text { (EHC } \\
\text { consultation) }\end{array}$ & $\begin{array}{l}\text { Female } \\
<25 \text { years }\end{array}$ & $\begin{array}{l}\text { FVU; } \\
\text { Postal return }\end{array}$ & Telephone & $\begin{array}{l}\text { GP or sexual } \\
\text { health clinician }\end{array}$ & $\begin{array}{l}\text { Offered: } 2904 \\
\text { Accepted: } 1348 \\
\text { (46.4\%) } \\
\text { Returned: } 236 \\
\text { (I7.6\%) }\end{array}$ \\
\hline $\begin{array}{l}\text { Emmerton et al, } \\
20 \mathrm{II}^{50} \\
\text { Australia }\end{array}$ & 4 months & $\begin{array}{l}\text { Opportunistic } \\
\text { (sexual health } \\
\text { consultation) }\end{array}$ & $\begin{array}{l}\text { Female } \\
\geq 16 \text { years }\end{array}$ & $\begin{array}{l}\text { FVU; } \\
\text { Postal return }\end{array}$ & $\begin{array}{l}\text { Telephone, } \\
\text { text message, } \\
\text { or letter }\end{array}$ & $\begin{array}{l}\text { GP or sexual } \\
\text { health clinician }\end{array}$ & $\begin{array}{l}\text { Distributed: } 156 \\
\text { Returned: } 18 \\
\text { (I2\%) }\end{array}$ \\
\hline $\begin{array}{l}\text { Currie et al, } \\
2012^{51} \\
\text { Australia }\end{array}$ & 4 weeks & $\begin{array}{l}\text { Opportunistic } \\
\text { (sexual health } \\
\text { consultation) }\end{array}$ & $16-30$ years & $\begin{array}{l}\text { FVU; } \\
\text { Returned } \\
\text { to issuing } \\
\text { pharmacy }\end{array}$ & Telephone & $\begin{array}{l}\text { GP or sexual } \\
\text { health clinician }\end{array}$ & $\begin{array}{l}\text { Distributed: } 979 \\
\text { Returned: } 900 \\
(91.9 \%)\end{array}$ \\
\hline $\begin{array}{l}\text { Habel et al, } \\
2015^{52} \\
\text { USA }\end{array}$ & 17 months & $\begin{array}{l}\text { Opportunistic } \\
\text { (EHC } \\
\text { consultation) }\end{array}$ & $\geq 18$ years & $\begin{array}{l}\text { FVU; } \\
\text { Left with } \\
\text { pharmacy- } \\
\text { based clinic }\end{array}$ & NR & NR & $\begin{array}{l}\text { Distributed: } 38 \\
\text { Returned: } 38 \\
(100 \%)\end{array}$ \\
\hline $\begin{array}{l}\text { Debattista et al, } \\
2016^{53} \\
\text { Australia }\end{array}$ & 9 months & Population & NR & $\begin{array}{l}\text { FVU; } \\
\text { Postal return }\end{array}$ & $\begin{array}{l}\text { Telephone, } \\
\text { text message, } \\
\text { or letter }\end{array}$ & $\begin{array}{l}\text { Contact tracing } \\
\text { support officer }\end{array}$ & $\begin{array}{l}\text { Distributed: } 109 \\
\text { Returned: } 43 \\
(39.4 \%)\end{array}$ \\
\hline \multicolumn{8}{|l|}{ Qualitative } \\
\hline $\begin{array}{l}\text { Author, year } \\
\text { country }\end{array}$ & Participants & \multicolumn{2}{|l|}{ Interview style } & \multicolumn{4}{|c|}{ Acceptability or barriers in pharmacy-based screening } \\
\hline $\begin{array}{l}\text { Taylor et al, } \\
2007^{54}\end{array}$ & $\begin{array}{l}\text { Pharmacists } \\
(\mathrm{n}=25)\end{array}$ & \multirow{2}{*}{\multicolumn{2}{|c|}{ Written questionnaire }} & \multirow{2}{*}{\multicolumn{4}{|c|}{$\begin{array}{l}\text { Pharmacists: } 84 \% \text { would support pharmacy-based chlamydia screening as } \\
\text { it would enhance their role. Training and incentives should be provided } \\
\text { Women: } 76 \% \text { would accept chlamydia screening from pharmacy due to } \\
\text { accessibility, anonymity, and affordability. The majority had concerns } \\
\text { about the level of privacy in a pharmacy setting }\end{array}$}} \\
\hline Australia & $\begin{array}{l}\text { Women aged } \\
\text { I8-29 } \\
\text { years }(n=50)\end{array}$ & & & & & & \\
\hline $\begin{array}{l}\text { Thomas et al, } \\
2009^{55}\end{array}$ & $\begin{array}{l}\text { Pharmacists } \\
(n=26)\end{array}$ & \multicolumn{2}{|c|}{ Written questionnaire $(n=26)$} & \multicolumn{4}{|c|}{$\begin{array}{l}\text { Pharmacists were keen to provide new services but made risk } \\
\text { assessments on who should be offered tests based on consumers' }\end{array}$} \\
\hline England & & \multicolumn{2}{|c|}{ Verbal in-depth interview $(n=12)$} & \multicolumn{4}{|c|}{$\begin{array}{l}\text { background (ethnicity, age, education) and type of relationship they } \\
\text { perceived them to be in }\end{array}$} \\
\hline $\begin{array}{l}\text { Dabrera et al, } \\
20 \mathrm{I}^{20} \\
\text { England }\end{array}$ & $\begin{array}{l}\text { Pharmacists } \\
(n=10)\end{array}$ & \multicolumn{2}{|c|}{ Semi-structured interview } & \multicolumn{4}{|c|}{$\begin{array}{l}\text { Pharmacists were supportive of chlamydia testing from pharmacies. Many } \\
\text { said they only offered a chlamydia test to women who requested EHC }\end{array}$} \\
\hline
\end{tabular}


Table 4 (Continued)

\begin{tabular}{|c|c|c|c|c|c|c|c|}
\hline \multicolumn{8}{|c|}{ Gonorrhea screening studies } \\
\hline $\begin{array}{l}\text { Author, year } \\
\text { country }\end{array}$ & Time frame & $\begin{array}{l}\text { Recruitment } \\
\text { approach }\end{array}$ & $\begin{array}{l}\text { Consumer } \\
\text { eligibility }\end{array}$ & $\begin{array}{l}\text { Type of } \\
\text { specimen; } \\
\text { method } \\
\text { of sample } \\
\text { return }\end{array}$ & $\begin{array}{l}\text { Result } \\
\text { notification }\end{array}$ & $\begin{array}{l}\text { Treating } \\
\text { health } \\
\text { professional }\end{array}$ & Uptake \\
\hline $\begin{array}{l}\text { Bloomfield } \\
\text { et al, } 2002^{46} \\
\text { USA }\end{array}$ & 2 weeks & Population & NR & $\begin{array}{l}\text { FVU; } \\
\text { Postal return }\end{array}$ & Telephone & $\begin{array}{l}\text { Pharmacist, GP, } \\
\text { or sexual health } \\
\text { clinician }\end{array}$ & $\begin{array}{l}\text { Distributed: } 209 \\
\text { Returned: } 80 \\
(38 \%)\end{array}$ \\
\hline $\begin{array}{l}\text { Habel et al, } \\
2015^{52} \\
\text { USA }\end{array}$ & 17 months & $\begin{array}{l}\text { Opportunistic } \\
\text { (EHC } \\
\text { consultation) }\end{array}$ & $\geq 18$ years & $\begin{array}{l}\text { FVU: } \\
\text { Left with } \\
\text { pharmacy- } \\
\text { based clinic }\end{array}$ & NR & NR & $\begin{array}{l}\text { Distributed: } 38 \\
\text { Returned: } 38 \\
(100 \%)\end{array}$ \\
\hline \multicolumn{8}{|c|}{ Syphilis screening studies } \\
\hline $\begin{array}{l}\text { Buchanan et al, } \\
2014-2015^{56} \\
\text { England }\end{array}$ & 9 months & $\begin{array}{l}\text { Population and } \\
\text { opportunistic } \\
\text { (needle exchange } \\
\text { and opiate } \\
\text { substitution } \\
\text { therapy) }\end{array}$ & NR & $\begin{array}{l}\text { Dry blood } \\
\text { spot; } \\
\text { Left with } \\
\text { pharmacist } \\
\text { to send to } \\
\text { Manchester } \\
\text { Royal Infirmary } \\
\text { for testing }\end{array}$ & $\begin{array}{l}\text { Automated } \\
\text { email }\end{array}$ & $\begin{array}{l}\text { Local sexual } \\
\text { health service }\end{array}$ & Completed: 88 \\
\hline \multicolumn{8}{|c|}{ Hepatitis B virus screening studies } \\
\hline $\begin{array}{l}\text { The Hepatitis C } \\
\text { Trust, } 2009^{57} \\
\text { England }\end{array}$ & 3 months & $\begin{array}{l}\text { Population and } \\
\text { opportunistic } \\
\text { (conversation } \\
\text { and educational } \\
\text { material to } \\
\text { identified at-risk } \\
\text { consumers) }\end{array}$ & $\geq 18$ years & $\begin{array}{l}\text { Dry blood } \\
\text { spot; } \\
\text { NR }\end{array}$ & $\begin{array}{l}\text { In person, } \\
\text { pharmacist-led }\end{array}$ & GP & Completed: 234 \\
\hline $\begin{array}{l}\text { Buchanan et al, } \\
2014-2015^{56} \\
\text { England }\end{array}$ & 9 months & $\begin{array}{l}\text { Population and } \\
\text { opportunistic } \\
\text { (needle exchange } \\
\text { and opiate } \\
\text { substitution } \\
\text { therapy) }\end{array}$ & NR & $\begin{array}{l}\text { Dry blood } \\
\text { spot; } \\
\text { Left with } \\
\text { pharmacist } \\
\text { to send to } \\
\text { Manchester } \\
\text { Royal Infirmary } \\
\text { for testing }\end{array}$ & $\begin{array}{l}\text { Automated } \\
\text { email }\end{array}$ & Hepatology team & Completed: 88 \\
\hline \multicolumn{8}{|c|}{$\begin{array}{l}\text { HIV screening studies } \\
\text { Quantitative }\end{array}$} \\
\hline $\begin{array}{l}\text { Calderon et al, } \\
2009-2011^{58} \\
\text { USA }\end{array}$ & 9 months & Population & $\begin{array}{l}\geq 13 \text { years } \\
\text { English- or } \\
\text { Spanish- } \\
\text { speaking }\end{array}$ & $\begin{array}{l}\text { Oral fluid } \\
\text { specimen; } \\
\mathrm{N} / \mathrm{A} \text { as } \\
\text { diagnosis } \\
\text { performed } \\
\text { on-site }\end{array}$ & $\begin{array}{l}\text { In person, by } \\
\text { a public health } \\
\text { advocate }\end{array}$ & HIV specialist & $\begin{array}{l}\text { Offered: } 2805 \\
\text { Accepted: } 2030 \\
(72.4 \%)\end{array}$ \\
\hline $\begin{array}{l}\text { Weidle et al, } \\
2011-2013^{59} \\
\text { USA }\end{array}$ & 2 years & Population & NR & $\begin{array}{l}\text { Oral fluid } \\
\text { specimen; } \\
\text { N/A as } \\
\text { diagnosis } \\
\text { performed } \\
\text { on-site }\end{array}$ & $\begin{array}{l}\text { In person, by } \\
\text { pharmacist, } \\
\text { retail clinic } \\
\text { staff, or on-site } \\
\text { representative } \\
\text { of local health } \\
\text { department }\end{array}$ & NR & $\begin{array}{l}\text { Completed: } \\
1540\end{array}$ \\
\hline
\end{tabular}


Table 4 (Continued)

\begin{tabular}{|c|c|c|c|}
\hline \multicolumn{4}{|l|}{ Qualitative } \\
\hline $\begin{array}{l}\text { Author, year } \\
\text { country }\end{array}$ & Participants & Interview style & Acceptability or barriers in pharmacy-based screening \\
\hline $\begin{array}{l}\text { Gorostiza et al, } \\
2009-2010^{60} \\
\text { Spain }\end{array}$ & $\begin{array}{l}\text { Consumers } \\
\text { of pharmacy- } \\
\text { accessed rapid } \\
\text { HIV antibody } \\
\text { screening tests } \\
(n=806)\end{array}$ & Survey & $\begin{array}{l}\text { Convenience, speed of testing, and accessibility of community pharmacies } \\
\text { were identified as the main motivators for consumers to accept the test }\end{array}$ \\
\hline $\begin{array}{l}\text { Darin et al, } \\
2010-2011^{61}\end{array}$ & $\begin{array}{l}\text { Adult consumers } \\
\text { presenting to }\end{array}$ & Written survey & $\begin{array}{l}\text { Among those who were undecided }(n=36 ; 9.5 \%) \text { or uninterested }(n=209 \text {; } \\
55 \%) \text { in pharmacy-based HIV screening, the main deterrents were the }\end{array}$ \\
\hline USA & $\begin{array}{l}\text { a community } \\
\text { pharmacy } \\
\text { prescription } \\
\text { counter }(n=380)\end{array}$ & & $\begin{array}{l}\text { lack of perceived risk or already understanding their HIV status. Main } \\
\text { facilitators were confidentiality, screening time of }<20 \text { minutes, use of a } \\
\text { private counseling room, and free screening }\end{array}$ \\
\hline
\end{tabular}

Abbreviations: NR, not reported; FVU, first void urine; GP, general practitioner; EHC, emergency hormonal contraception; N/A, not applicable; HIV, human immunodeficiency virus.

that addresses necessary criteria for consideration when conceptualizing a targeted approach to chlamydia screening. For effective implementation, issues around confidentiality in a community pharmacy setting, pharmacy reimbursement, and the cost of chlamydia screening must be considered for individual countries and unique health settings.

\section{Gonorrhea}

\section{Background}

WHO estimates that there are $\sim 78$ million new cases of gonorrhea every year worldwide, with the greatest incidence seen in the Western Pacific, South-East Asian and African regions (Figure 1). ${ }^{1}$ Recent global prevalence is estimated to be $0.6 \%$ of men and $0.8 \%$ of women. ${ }^{23}$ Those at increased risk for gonorrheal infection include sexually active females and males aged 15-29 years, those with multiple recent sexual partners or new sexual partners, men who have sex with men, and presence of other STIs such as chlamydia. ${ }^{62}$

Gonorrhea is an issue of global public health concern; as well as being asymptomatic in most infected consumers, antibiotic-resistant strains of Neisseria gonorrhoeae are emerging in recent years, reducing the effectiveness of some antibiotic treatment. The Centers for Disease Control and Prevention (CDC) estimate that, of the 820,000 new gonorrhea infections that occur each year in the USA, $\sim 246,000$ show resistance to at least one antibiotic. ${ }^{63}$ While efforts are being made to identify and develop new antibiotics that can treat gonorrhea, the CDC has compiled a set of guidelines as part of their Solutions Initiative to reduce drug-resistant gonorrhea ${ }^{63}$ The guidelines recommend expanding the availability of resistance testing, reducing the time taken for health providers to receive test results to ensure timely treatment, and identify and manage sexual partners promptly to minimize the spread of resistance. ${ }^{63}$

\section{Current practice}

Screening for gonorrhea involves testing by using NAAT for all specimens except urethral swabs, which rely on cultures for diagnosis (Table 5). ${ }^{6}$ Culture samples can be collected from all specimens except first void urine (FVU). ${ }^{6}$ Although not as sensitive as NAAT, they can be used to determine the antibiotic sensitivity of the organism to guide treatment. ${ }^{6}$

WHO guidelines for gonorrheal treatment recommend that local resistance data should guide treatment choice; In general, first-line choice when antibiotic susceptibility information is unknown should involve a combination therapy with two antibacterial medications of differing mechanisms of action. ${ }^{64}$ This strategy improves treatment effectiveness and can slow down the occurrence of antibiotic resistance in $N$. gonorrhoeae.$^{65}$ Recommended dual therapy is either ceftriaxone ( $250 \mathrm{mg}$ intramuscular single dose) plus azithromycin (1 $\mathrm{g}$ oral single dose), or cefixime (400 mg oral single dose) plus azithromycin ( $1 \mathrm{~g}$ oral single dose $).{ }^{64} \mathrm{As}$ azithromycin treats chlamydial infections in this dose and delivery, there is an additional benefit of simultaneously treating a chlamydial coinfection. ${ }^{64}$ Coinfection with chlamydia has been demonstrated to be as high as almost $50 \%$ in gonorrhea-positive consumers. ${ }^{66,67}$ When specific antibiotic susceptibility is known, this should be used to determine appropriate treatment. ${ }^{64}$ 
Table 5 Specimens collected for gonorrhea screening

\begin{tabular}{|c|c|c|}
\hline Specimen Testing protocol & & hen collected \\
\hline First void urine & $0^{2}$ & MSM \\
\hline NAAT & \& & Only if endocervical swab or vaginal swab cannot be taken, but not as sensitive as vaginal swab \\
\hline Anorectal swab & $\sigma^{7}$ & All MSM \\
\hline NAAT \pm culture & Q & If patient has had anal sex or has anorectal symptoms \\
\hline Pharyngeal swab & $\sigma^{7}$ & All MSM \\
\hline NAAT \pm culture & ९ & If patient has had oral sex \\
\hline $\begin{array}{l}\text { Endocervical swab } \\
\text { NAAT } \pm \text { culture }\end{array}$ & q & Collected upon examination if discharge or dysuria is present \\
\hline $\begin{array}{l}\text { Vaginal swab } \\
\text { NAAT } \pm \text { culture }\end{array}$ & Q & Collected when no examination \\
\hline $\begin{array}{l}\text { Urethral swab } \\
\text { Culture }\end{array}$ & $\sigma^{2}$ & When discharge present \\
\hline
\end{tabular}

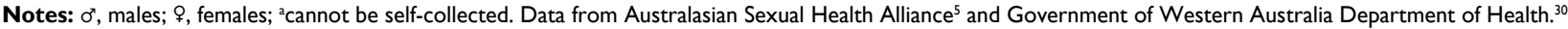
Abbreviations: NAAT, Nucleic Acid Amplification Test; MSM, men who have sex with men.

\section{Role of the pharmacist}

Gonorrhea screening kits can be purchased from selected pharmacies in the USA, UK, and Australia, with some UK pharmacies also providing free urine-based screening kits over the counter. ${ }^{40-43}$

Two relevant studies were identified that assessed the feasibility of pharmacy-based gonorrhea screening; both were conducted in the US (Table 4). The timescales of interventions ranged from 2 weeks to 17 months, and the number of completed screenings varied from 38 to 80 . Both the studies concluded that gonorrhea screening from community pharmacies is feasible, particularly when offered in conjunction with chlamydia screening. Almost all opportunistically targeted consumers felt that pharmacists should be involved in STI screening, with most reporting satisfaction with their experience. When population-based screening was studied, pharmacists felt that consumers chose to collect screening kits because pharmacies could provide convenient access to a relatively anonymous service.

Bloomfield et $\mathrm{al}^{46}$ trialed a population-based screening approach that additionally targeted advertising toward the local men who have sex with men (MSM) subgroup. Ninetyfive percent of returned specimens belonged to MSM, suggesting that advertising aimed toward at-risk groups could be successful, but trialing over a longer time frame is needed before any conclusions can be made. Habel et al trialed opportunistic targeting of at-risk people; they provided a voucher for gonorrhea screening to anyone who purchased EHC during the study period. ${ }^{52}$ Screening uptake was low, which authors suggested could be due to the lack of both advertising and pharmacist-driven discussion about screening.

Both the studies only offered self-collection of urine as a specimen, regardless of gender. A systematic review and meta-analysis by Lunny et al found that, when comparing self-sampled specimens with the equivalent cliniciancollected samples, sensitivity of screening urine specimens from asymptomatic women was low. This finding supports CDC guideline recommendations that urine specimens should not be collected to screen asymptomatic women for gonorrhea due to relatively low sensitivity, with preference given to self-collected vaginal swabs. ${ }^{65,68}$ To date, no trials have been performed to evaluate the effectiveness of using vaginal swabs to screen women for gonorrhea through a community pharmacy. In the studies that collected urine samples, neither Bloomfield et al nor Habel et al retested self-collected samples or gathered clinician-collected samples to verify the accuracy of results; hence, it is possible that similarly low sensitivity was observed, and by extension some gonorrheapositive consumers may not have been diagnosed during the study period. Consequently, the infection would remain untreated and undiagnosed for an indeterminate amount of time, or any future symptoms may be dismissed due to an incorrect belief that they are gonorrhea-negative.

As well as potential sensitivity issues, urine-based sampling can introduce further issues with storage and handling. While urine samples can maintain accuracy when stored at room temperature for up to 7 days, locations with higher ambient temperatures would need to assess whether stricter storage requirements are necessary to maintain stability. ${ }^{46} \mathrm{In}$ addition, Bloomfield et al noted that almost $4 \%$ of returned urine samples were lost due to improper sealing of the collection cup. ${ }^{46}$ Vaginal swabs offer further advantages as they do not have the leakage problems associated with transporting urine samples. ${ }^{69}$ If vaginal swab sampling is used, there is potential for gonorrhea and chlamydia screening initiatives to merge. Because NAAT testing (the preferred diagnostic 
test for both infections) has high sensitivity and specificity when performed on urine samples from men and vaginal swab specimens from women - and there is an elevated risk of chlamydia coinfection in gonorrhea-positive consumers - this could prove to be both beneficial and cost-effective.

\section{Summary}

There is some evidence that pharmacists can contribute to gonorrhea screening, when performed in conjunction with chlamydia screening. Current WHO recommendations suggest that collected samples should be restricted to urine specimens for men and vaginal swabs for women for optimal sensitivity, but use of vaginal swabs require further exploration in a pharmacy setting.

\section{Syphilis \\ Background}

According to WHO, $~ 5.6$ million new syphilis infections occur globally every year and 18 million established infections at any one time. ${ }^{1,70}$ The most recent estimated global prevalence indicated that $0.48 \%$ of men and $0.5 \%$ of women aged 15-49 years have a syphilis infection. ${ }^{23}$ The highest prevalence for both men and women is noted in developing countries, particularly in African nations (Figure 1). ${ }^{23}$ Syphilis most commonly affects MSM, men aged 15-54 years, and

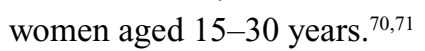

When first infected with syphilis, the bacteria enters the body and usually leaves behind at least one small, painless lesion called a chancre that heals on its own within weeks; this is known as primary syphilis. ${ }^{72} \mathrm{~A}$ few weeks later, secondary syphilis may be seen; typically a rash that can cover the entire body. ${ }^{72}$ It can be accompanied by wart-like oral or genital lesions and flu-like symptoms. ${ }^{72}$ This stage can last anywhere from a few weeks to a year; during which the infection becomes latent; with no obvious signs and/or symptoms. ${ }^{72}$ The infection can thus remain latent permanently or could progress to tertiary syphilis that leads to a range of neurological or cardiovascular problems. ${ }^{72}$ Pregnant women can pass syphilis to their baby through the placenta or during childbirth, greatly increasing the risk of miscarriage, stillbirth, or neonatal death. ${ }^{72}$ Most newborns will not display symptoms but untreated syphilis can lead to deafness, tooth deformities, and a collapsed nasal bridge. ${ }^{72}$ In 2012, $~ 350,000$ adverse pregnancy outcomes (infection, low birth weight, stillbirth, and neonatal death) were attributed to syphilis. ${ }^{70}$ It is the consequences of tertiary and congenital syphilis that contribute to the public health concern associated with syphilis. ${ }^{72}$ In addition, chancres bleed easily so can increase the risk of contracting HIV by an estimated two to five times. ${ }^{72}$

\section{Current practice}

When screening for syphilis, a whole blood specimen is taken and usually screened with an enzyme immunoassay, although agglutination assays and hemagglutination assays can be used. ${ }^{7}$ If reactive, or if the consumer has previously had a treated syphilis infection, rapid plasma reagin is used to confirm. ${ }^{7}$ Screening between one and four times per year is recommended for MSM; HIV-positive MSM should be screened up to four times per year or at every viral load monitor. ${ }^{7}$ Pregnant women should be tested at least once during antenatal appointments, with a repeat screen performed in late pregnancy if she is at risk of infection or reinfection. ${ }^{7}$ First-line treatment is benzathine penicillin ( $1.8 \mathrm{~g}$ intramuscular single dose) in all populations, with doxycycline (100 mg orally twice a day for 14 days) used when penicillin allergy exists. ${ }^{7}$

\section{Role of the pharmacist}

Syphilis screening kits can be purchased from selected community pharmacies in the USA and the UK. ${ }^{41,73}$ In these kits, the consumers self-collect the sample by using a thin lancet on their fingertip to draw blood, and the results will be available on the test cassette within 15 minutes. Syphilis screening kits have a high level of accuracy; they report the sensitivity and specificity at over $99 \%$ and come with a control line to demonstrate validity. ${ }^{74,75}$ These screening kits have not been trialed for accuracy or consumer satisfaction.

Our literature search identified one study that trialed pharmacy-based syphilis screening, in England (Table 4). Recruitment was both opportunistic and population-based; pharmacists and pharmacy staff targeted anyone participating in the needle exchange program or opiate substitution therapy, while local advertising informed the general population of the screening initiative. ${ }^{56}$ Pharmacists were trained in conducting a dry blood spot sampling method, counseling consumers, and collecting samples, which were then sent to Manchester Royal Infirmary for testing. ${ }^{56}$ Out of 88 participants who were screened for syphilis, 72 were recruited opportunistically by pharmacists; most participants identified themselves as intravenous drug users. ${ }^{56}$

Buchanan et $\mathrm{al}^{56}$ concluded that dry blood spot syphilis screening from a community pharmacy is both accessible and acceptable to consumers. ${ }^{56}$ However, it involved a high level of commitment and training from pharmacists, which can pose problems. Logistically, it would require every 
pharmacist in participating pharmacies to complete the necessary training, so that screening is readily available during all opening hours. Locums working in participating pharmacies would need to be trained to continue the service when the usual pharmacist is away. Dry blood spot sampling also involves a high level of risk as it requires the pharmacist to handle whole blood from consumers who potentially carry blood-borne infections.

\section{Summary}

There is not enough evidence to date that pharmacist-led syphilis screening is feasible. While there may be potential for an initiative to be successful, more information is needed in a wider variety of settings with targeted recruitment of atrisk populations. If syphilis screening through community pharmacies is justified, consideration should be given to alternative methods of screening that reduce the risks to the pharmacist, such as over-the-counter screening kits.

\section{HBV}

\section{Background}

Globally, there are $\sim 257$ million people infected with HBV, with the highest prevalence observed in adult populations in the Western Pacific (6.2\%) and African (6.1\%) regions. ${ }^{76}$ Although sexual transmission is not the most common route of infection, it can occur through exchange of or mucosal exposure to infected bodily fluid. ${ }^{76}$

$\mathrm{HBV}$ is potentially life-threatening, causing chronic infection and leading to a high risk of death from cirrhosis or liver cancer; in 2015, 887,000 people were affected from HBVrelated complications. ${ }^{76}$ Chronic liver infections are common in infants and children who became infected in utero. ${ }^{76}$

\section{Current practice}

HBV can only be differentiated from other types of viral hepatitis by laboratory confirmation. ${ }^{76} \mathrm{~A}$ blood sample is tested for the presence of the HBV surface antigen HBsAg, by using enzyme immunoassay techniques. ${ }^{76,77}$ Screening is recommended in pregnant women, adults at increased risk of transmission, and people who are hepatitis C-positive or HIV-positive due to the increased risk of coinfection. ${ }^{77}$

There is no cure for HBV; treatment in acute infections is aimed at relieving symptoms and replacing fluids lost through vomiting and diarrhoea. ${ }^{76}$ Medication can be used to manage chronic infection, but treatment is complex and case-specific, depending on the duration of infection, presence of symptoms, and complications arising from chronic infection. ${ }^{78}$ Life-long antiviral medication such as tenofovir can be used to slow down the ability of the virus to multiply in chronic $\mathrm{HBV}$ infections. ${ }^{76,78}$

\section{Role of the pharmacist}

Rapid screening kits to test for HBsAg are available, allowing consumers to self-collect their own blood samples directly into a test cassette. ${ }^{79}$ Results are visually seen on the test cassette within 15 minutes, and the screening kit offers a sensitivity and specificity of $99 \% .{ }^{79}$ High accuracy, self-collection, and privacy of testing make it an attractive screening option to some high-risk consumers.

Our literature review shows that pharmacist-led HBV screening studies have been trialed in England (Table 4). Length of interventions varied from 3 to 9 months, and 234 and 80 screenings were completed, respectively. Both the studies concluded that HBV screening is feasible in a community pharmacy setting, and combining it with hepatitis $\mathrm{C}$ screening adds cost-effectiveness. Pharmacists in The Hepatitis C Trust initiative found their whole-day training session to be comprehensive and helpful, with several displaying enthusiasm for the "exciting initiative. ${ }^{\prime 57}$ Likewise, post-participation consumer feedback was very positive; out of the 19 consumers who completed the survey, 18 indicated that they preferred screening from a pharmacy setting as opposed to a GP surgery. ${ }^{57}$ All surveyed consumers found the service convenient and would recommend it to at-risk friends. ${ }^{57}$ Neither pharmacist nor consumer satisfaction was reported by Buchanan et al. ${ }^{56}$

The same concerns with respect to dry blood spot syphilis screening apply in this context; HBV screening using dry blood spot sampling requires a high level of pharmacist involvement, and the exposure to potentially infected blood samples poses a risk. The protocol trialed by The Hepatitis C Trust placed even further responsibilities onto the pharmacist. In addition to involvement in pre- and posttest counseling, sample collection, and handling, pharmacists were required to discourage the "worried well" with no risk factors from being screened, follow up with each screened consumer to ensure they returned to the pharmacy for their results, and provide tailored education to HBV-negative or HBV-positive consumers. The report did not indicate how much time pharmacists spent following up with consumers, the number of consumers who failed to return in the required 2-3 weeks for their results, or pharmacists' perspectives of the time taken to comprehensively manage consumers, but these factors must be considered when assessing the feasibility of this initiative in daily practice. 
However, The Hepatitis C Trust was more stringent on minimizing the pharmacists' risk of personal injury. The pharmacist training session was twice as long as the equivalent in the study conducted by Buchanan et al, ${ }^{56}$ and all participating pharmacists were required to complete a super accelerated course of $\mathrm{HBV}$ vaccinations if unvaccinated and provide proof that adequate antibody levels had been achieved prior to participation. These two requirements reduced the level of personal risks that participating pharmacists would be exposed to.

\section{Summary}

There is some evidence that pharmacists can have an important role in assisting at-risk consumers to understand their risk factors, provide a relatively anonymous route for HBV screening, and assist in specimen collection, diagnosis, and treatment coordination. However, pharmacists may not have time to perform thorough HBV screening, and dry blood spot sampling still poses a significant health risk. Future trials should investigate a less time-intensive and risky pharmacist-led protocol, such as provision of over-the-counter screening kits.

\section{HIV}

\section{Background}

Latest WHO estimates indicate that 37 million people are infected with HIV globally; of these, nearly half are unaware of their HIV status. ${ }^{80}$ While the recent HIV response to reduce global incidence has been effective in some countries, incidence continues to rise in areas such as sub-Saharan Africa (Figure 2) where $\sim 1$ in 25 adults are infected. ${ }^{80,81} \mathrm{MSM}$, transgender women, and residents of (or travelers to) countries with high incidence of HIV are in particular at risk of sexually transmitted HIV. ${ }^{82}$

HIV infection causes a chronic immune deficiency that usually first presents as a flu-like illness 2 weeks after exposure. ${ }^{12}$ While currently incurable, if detected early, then life-long combination antiretroviral therapy (ART) can be used to lower the viral load, prevent HIV transmission, and prevent progression from HIV to acquired immunodeficiency syndrome (AIDS). ${ }^{12}$ Approximately 1 million people died of HIV-related illnesses in 2016; the high mortality associated with opportunistic infections in HIV-positive people makes HIV a serious global public health threat. ${ }^{80}$

\section{Current practice}

Testing for HIV is a two-step process. The first step is to test for HIV-specific proteins (antibodies or antigens) in the plasma, serum, whole blood, or oral fluid. The two main types of HIV self-screening kits available from pharmacies and/ or online involve either a finger stick/venous whole blood $(\sim 5 \mu \mathrm{L})$ sample, or oral saliva test conducted by swabbing

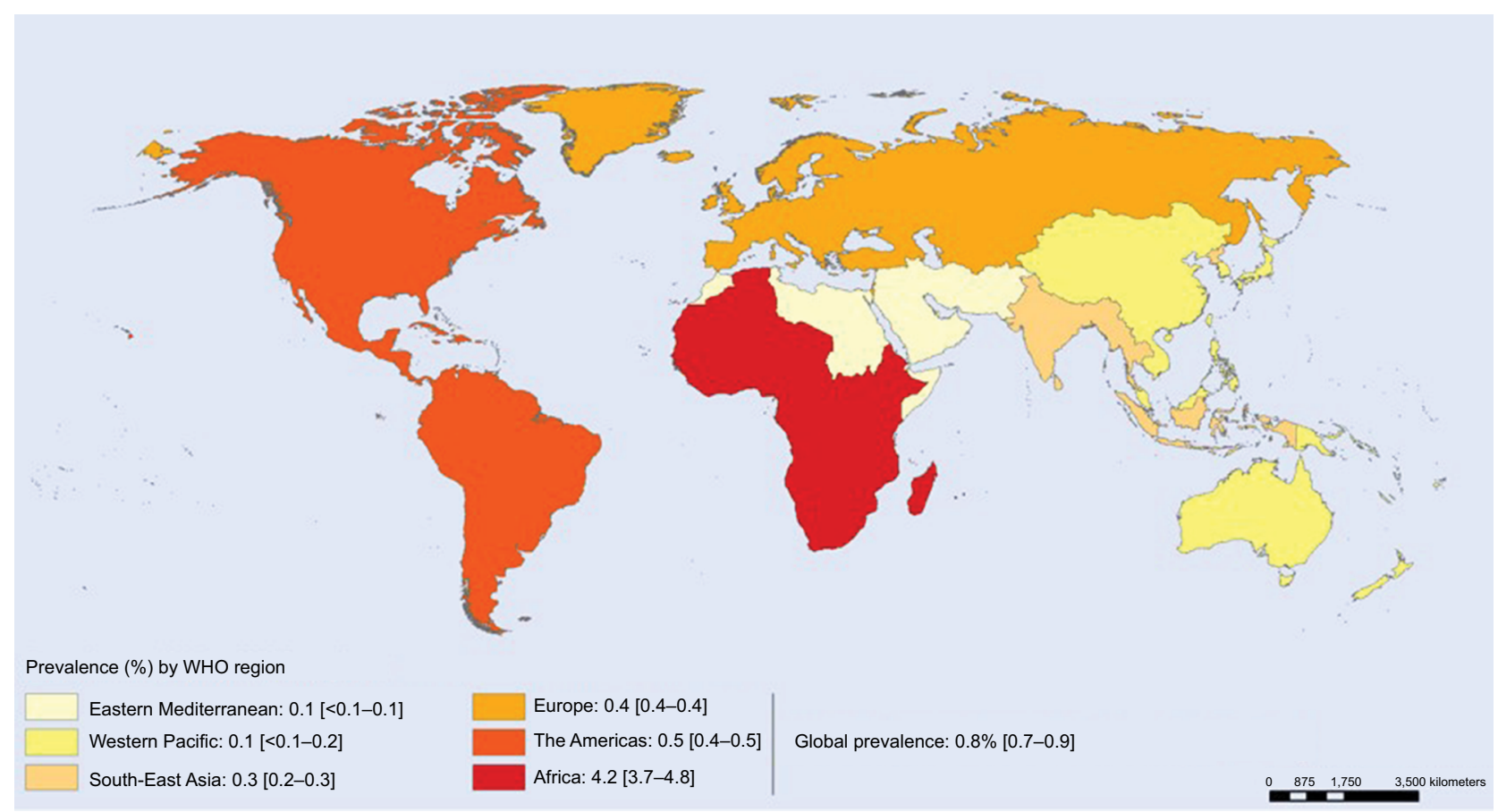

Figure 2 Prevalence of HIV in 15-49 year olds, by WHO region.

Note: Reprinted from World Health Organization [webpage on the Internet]. HIV/AIDS. Available from: http://www.who.int/gho/hiv/en/.81

Abbreviations: HIV, human immunodeficiency virus; WHO, World Health Organization. 
the upper and lower gum line. The results of both these tests take about 20-40 minutes. If the HIV self-screen results in a positive test, then the individual needs to undergo a second test called a Western blot, which involves skilled staff and equipment. Only when both the tests are positive, it is almost 99\% certain that the patient is infected with HIV. The CDC advises HIV screening for sexually active MSM every 3-6 months, annual screening for anyone who has unprotected sexual intercourse or shares injection drug equipment, and one-off screening for all pregnant women. ${ }^{83}$ In addition, they recommend that all adolescents and adults aged between 13 and 64 years should be tested at least once for HIV. ${ }^{83}$

Although there is no cure for HIV, lifelong ART medication tailored to the individual can be used effectively to control the virus. There are five main groups of ART medications, which target different stages of the virus lifecycle; combination ART uses multiple medications from at least two of these groups and is taken orally once or twice a day. ${ }^{84}$ AIDS-related illnesses, transmission rates, hospital admissions, and mortality rates can all be reduced with early ART intervention. ${ }^{84}$ Depending on location and available health services, high-risk HIV-negative consumers may be able to access pre-exposure prophylaxis (PrEP), a combination ART that, when taken daily, can help prevent HIV infection. ${ }^{85}$

\section{Role of the pharmacist}

As ART and PrEP are essential components of management and prevention, respectively, pharmacists can provide support with medication management and education. They are in an ideal position to identify and discuss any medication side effects and offer relief or refer to the treating health specialist. They can also identify the use of complementary medicines and ensure they are being used safely with no interaction. Most importantly, pharmacists are able to send prescription refill reminders and emphasize that ART and PrEP must be taken daily to be effective. ${ }^{84,85}$ Missed ART doses can lead to treatment resistance and missed PrEP can reduce effectiveness and potentially expose the consumer to infection, so medication compliance is crucial for success. ${ }^{84,85}$

We identified several pilot programs that assessed the feasibility of pharmacy-based HIV screening (Table 4). Two quantitative studies were conducted in the USA; timescales ranged from 9 months to 2 years, with 2030 and 1540 screenings performed, respectively. Calderon et $\mathrm{al}^{58}$ and Weidle et $\mathrm{al}^{59}$ concluded that HIV screening through a community pharmacy could be successfully implemented.

Both the studies screened oral fluid specimen rather than whole blood or blood products. Screening oral fluid greatly reduces the personal risk to the pharmacist; brief contact with saliva from an HIV-positive individual has been shown to be "insignificant" in HIV transmission, while contact with blood and semen greatly increases the risk of transmission. ${ }^{86}$

There are financial and logistical barriers that need to be overcome for successful implementation. The biggest barrier relates to remuneration for services, particularly considering that HIV screening is time-intensive; Weidle et al found that pharmacists spent an average of 10 and 21 minutes on non-reactive and reactive screens, respectively, for pre- and posttest consultations. ${ }^{59} \mathrm{HIV}$ screening and counseling is a skilled, highly involved, and time-consuming initiative for community pharmacists, so reimbursement for this service must be appropriate and sustainable. In addition, in countries where private counseling rooms are not mandatory, improving infrastructure to assure confidentiality can be expensive and may not be financially viable if remuneration is low.

\section{Summary}

There is some evidence that pharmacist-led HIV screening is possible, particularly through use of oral fluid specimens. However, a larger number of studies from a wider variety of settings is required before any conclusions can be reached. Consideration must be given to pharmacist reimbursement and infrastructure upgrades with respect to the unique healthcare system and pharmacy infrastructure of each country.

\section{Trichomoniasis and HSV}

There have been no studies to date that have examined the possibility of pharmacist-led screening for trichomoniasis or HSV.

Trichomoniasis can be detected in urine sample; hence, there is a potential for a pharmacy-based screening initiative, provided the sensitivity and specificity of the diagnostic test are high. ${ }^{8}$ However, pharmacist-led trichomoniasis screening would need to be assessed for feasibility before any conclusions can be made.

The CDC does not recommend screening for HSV; the most accurate diagnosis can be made by testing a swab sample taken from a symptomatic lesion or blister. ${ }^{87}$ Absence of symptoms is a key requirement of screening appropriateness; hence, we do not foresee any potential role for pharmacists in this scenario.

\section{Conclusion}

STI screening is a critical component of sexual health, ensuring that infections are detected as early as possible for prompt treatment and optimal health outcomes. Avenues for safe, accurate STI screening must be identified and supported, to increase detection in at-risk populations. Pharmacist-led 
screening has many benefits: sample collection is either nonor minimally invasive, some samples can be self-collected in the privacy of the consumers' home, and is more convenient than clinician-led testing. To be effective, pharmacist-led screening must also have demonstrated high sensitivity and specificity and must be acceptable to consumers. The role of pharmacists in providing sexual health screening can be as simple as providing an over-the-counter screening kit, or they can bear more responsibility with tasks such as recommending STI screening to at-risk consumers, collecting samples, educating consumers, referring to appropriate health services, and managing medications. Although some STIs have no or some evidence that pharmacists are well-placed to provide screening services, there is sufficient evidence that pharmacists can assume a more prominent role in screening for STIs such as chlamydia. However, further investigations are needed - particularly with respect to remuneration, infrastructure, screening accuracy, and pharmacist safety - before global recommendations can be made.

\section{Disclosure}

The authors report no conflicts of interest in this work.

\section{References}

1. World Health Organization [webpage on the Internet]. Sexually transmitted infections (STIs); 2016. Available from: http://www.who.int/ mediacentre/factsheets/fs110/en/. Accessed August 5, 2017.

2. healthdirect [webpage on the Internet]. Sexually transmitted infections (STI); 2016. Available from: https://www.healthdirect.gov.au/sexuallytransmitted-infections-sti. Accessed August 5, 2017.

3. Office on Women's Health [webpage on the Internet]. Sexually transmitted infections, pregnancy, and breastfeeding. Available from: https:// www.womenshealth.gov/a-z-topics/stis-pregnancy-and-breastfeeding. Accessed August 6, 2017.

4. World Health Organization [webpage on the Internet]. Sexual and reproductive health. Available from: http://who.int/reproductivehealth/ topics/rtis/en/. Accessed August 23, 2017.

5. Australasian Sexual Health Alliance [webpage on the Internet]. Australian STI Management Guidelines for Use in Primary Care: Chlamydia. Available from: http://www.sti.guidelines.org.au/sexually-transmissibleinfections/chlamydia\#management. Accessed August 5, 2017.

6. Australasian Sexual Health Alliance [webpage on the Internet]. Australian STI Management Guidelines for Use in Primary Care: Gonorrhoea. Available from: http://www.sti.guidelines.org.au/sexually-transmissibleinfections/gonorrhoea. Accessed August 5, 2017.

7. Australasian Sexual Health Alliance [webpage on the Internet]. Australian STI Management Guidelines for Use in Primary Care: Syphilis. Available from: http://www.sti.guidelines.org.au/sexually-transmissibleinfections/syphilis. Accessed August 5, 2017.

8. Australasian Sexual Health Alliance [webpage on the Internet]. Australian STI Management Guidelines for Use in Primary Care: Trichomoniasis. Available from: http://www.sti.guidelines.org.au/ sexually-transmissible-infections/trichomoniasis. Accessed August 5, 2017.

9. Australasian Sexual Health Alliance [webpage on the Internet]. Australian STI Management Guidelines for Use in Primary Care: Herpes. Available from: http://www.sti.guidelines.org.au/sexually-transmissibleinfections/herpes. Accessed August 5, 2017.
10. Australasian Sexual Health Alliance [webpage on the Internet]. Australian STI Management Guidelines for Use in Primary Care: Hepatitis B. Available from: http://www.sti.guidelines.org.au/sexuallytransmissible-infections/hepatitis-b. Accessed August 5, 2017.

11. Australasian Sexual Health Alliance [webpage on the Internet]. Australian STI Management Guidelines for Use in Primary Care: Genital Warts. Available from: http://www.sti.guidelines.org.au/ sexually-transmissible-infections/hpv. Accessed August 5, 2017.

12. Australasian Sexual Health Alliance [webpage on the Internet]. Australian STI Management Guidelines for Use in Primary Care: HIV. Available from: http://www.sti.guidelines.org.au/sexually-transmissibleinfections/hiv. Accessed August 5, 2017.

13. Centers for Disease Control and Prevention. Sexually transmitted infections in developing countries: current concepts and strategies on improving STI prevention, treatment, and control; 2008. Available from: http://siteresources.worldbank.org/INTPRH/Resources/STINoteFINAL26Feb08.pdf. Accessed August 6, 2017.

14. Giesecke J, editor. The epidemiology of vaccination. In: Modern Infectious Disease Epidemiology. 2nd ed. London: Hodder Arnold; 2002:226-258.

15. The Royal Children's Hospital Melbourne [webpage on the Internet]. Sexually transmitted infections (STIs). Available from: https://www. rch.org.au/clinicalguide/guideline_index/Sexually_transmitted_infections_STIs/.Accessed August 23, 2017.

16. Unemo M, Bradshaw CS, Hocking JS, et al. Sexually transmitted infections: challenges ahead. Lancet Infect Dis. 2017;17(8):e235-e279.

17. Chacko MR, von Sternberg K, Velasquez MM, Wiemann CM, Smith $\mathrm{PB}, \mathrm{DiClemente} \mathrm{R}$. Young women's perspective of the pros and cons to seeking screening for chlamydia and gonorrhea: an exploratory study. J Pediatr Adolesc Gynecol. 2008;21(4):187-193.

18. Eng TR, Butler WT, editors. The neglected health and economic impact of STDs. In: The Hidden Epidemic: Confronting Sexually Transmitted Diseases. Washington, DC: National Academies Press (US); 1997:28-68.

19. Low N, Broutet N, Adu-Sarkodie Y, Barton P, Hossain M, Hawkes S. Global control of sexually transmitted infections. Lancet Glob Health. 2006;368(9551):2001-2016.

20. Dabrera G, Pinson D, Whiteman S. Chlamydia screening by community pharmacists: a qualitative study. J Fam Plann Reprod Health Care. 2011;37(1):17-21.

21. Benrimoj SI, Frommer MS. Community pharmacy in Australia. Aust Health Rev. 2004;28(2):238-246.

22. Brabin L, Thomas G, Hopkins M, O'Brien K, Roberts SA. Delivery of chlamydia screening to young women requesting emergency hormonal contraception at pharmacies in Manchester, UK: a prospective study. BMC Womens Health. 2009;9(1):7.

23. Newman L, Rowley J, Vander Hoorn S, et al. Global estimates of the prevalence and incidence of four curable sexually transmitted infections in 2012 based on systematic review and global reporting. PLoS One. 2015;10(12):e0143304.

24. Lim MS, Goller JL, Guy R, et al. Correlates of Chlamydia trachomatis infection in a primary care sentinel surveillance network. Sexual Health. 2012;9(3):247-253.

25. Kalwij S, Macintosh M, Baraitser P. Screening and treatment of Chlamydia trachomatis infections. BMJ. 2010;340:c1915.

26. Lallemand A, Bremer V, Jansen K, et al. Prevalence of Chlamydia trachomatis infection in women, heterosexual men and MSM visiting HIV counselling institutions in North Rhine-Westphalia, Germany-should Chlamydia testing be scaled up? BMC Infect Dis. 2016;16(1):610.

27. Land J, Van Bergen J, Morre S, Postma M. Epidemiology of Chlamydia trachomatis infection in women and the cost-effectiveness of screening. Hum Reprod Update. 2009;16(2):189-204.

28. Melbourne Sexual Health Centre [webpage on the Internet]. STI Screening. Available from: http://www.mshc.org.au/HealthProfessional/ MSHCTreatmentGuidelines/STISCREENING\#.WeYKdjBLeUk. Accessed September 18, 2017.

29. World Health Organization [webpage on the Internet]. WHO guidelines for the treatment of Chlamydia trachomatis. 2016. Available from: http:// www.who.int/reproductivehealth/publications/rtis/chlamydia-treatmentguidelines/en/. Accessed August 17, 2017. 
30. Government of Western Australia Department of Health [webpage on the Internet]. Chlamydia - testing and clinical management. Available from: http://ww2.health.wa.gov.au/Articles/A_E/Chlamydia-testingand-clinical-management. Accessed October 2, 2017.

31. Workowski KA, Berman SM. Centers for Disease Control and Prevention sexually transmitted disease treatment guidelines. Clin Infect Dis. 2011;53(Suppl 3):S59-S63.

32. Nwokolo NC, Dragovic B, Patel S, William Tong CY, Barker G, Radcliffe K. 2015 UK national guideline for the management of infection with Chlamydia trachomatis. Int J STD AIDS. 2016;27(4):251-267.

33. Chacko MR, von Sternberg K, Velasquez MM, Wiemann CM, Smith $\mathrm{PB}$, DiClemente R. Young women's perspective of the pros and cons to seeking screening for chlamydia and gonorrhea: an exploratory study. J Pediatr Adolesc Gynecol. 2008;21(4):187-193.

34. Balfe M, Brugha R. What prompts young adults in Ireland to attend health services for STI testing? BMC Public Health. 2009;9(1):311.

35. Ford CA, Jaccard J, Millstein SG, Viadro CI, Eaton JL, Miller WC Young adults' attitudes, beliefs, and feelings about testing for curable STDs outside of clinic settings. J Adolesc Health. 2004;34(4): 266-269.

36. Balfe M, Brugha R, O'Connell E, McGee H, O'Donovan D, Vaughan D. Why don't young women go for Chlamydia testing? A qualitative study employing Goffman's stigma framework. Health Risk Soc. 2010;12(2):131-148.

37. Kong F, Guy RJ, Hocking JS, et al. Australian general practitioner chlamydia testing rates among young people. Med JAust. 2011;194(5): 249-252.

38. St. Lawrence JS, Montaño DE, Kasprzyk D, Phillips WR, Armstrong $\mathrm{K}$, Leichliter JS. STD screening, testing, case reporting, and clinical and partner notification practices: a national survey of US physicians. Am J Public Health. 2002;92(11):1784-1788.

39. McNulty CA, Freeman E, Howell-Jones R, et al. Overcoming the barriers to chlamydia screening in general practice - a qualitative study Fam Pract. 2010;27(3):291-302.

40. freetest.me [webpage on the Internet]. Local chlamydia/STI testing services. Available from: https://www.freetest.me/local/. Accessed September 2, 2017.

41. Target [webpage on the Internet]. STD tests: sexual health. Available from: https://www.target.com/c/sexual-health/std-tests/-/N5xu08Z4utbz. Accessed September 2, 2017.

42. Boots Web MD [webpage on the Internet]. STI tests. Available from: http://www.webmd.boots.com/sexual-conditions/guide/sti-tests Accessed September 2, 2017.

43. Fresh Therapeutics Compounding Pharmacy [webpage on the Internet] Sexually Transmitted Chlamydia and Gonorrhoea. Available from: http://www.freshtherapeutics.com.au/professional-services/risk-assessment-and-screening/sexually-transmitted-chlamydia-and-gonorrhoea/. Accessed September 2, 2017.

44. National Chlamydia Screening Programme. Information to support the commissioning of chlamydia screening in general practice and community pharmacies; 2014. Available from: https://www.gov.uk/ government/uploads/system/uploads/attachment_data/file/373205/ Developing_service_specifications_for_chlamydia_testing_and_treatment_in_GP_and_CommPharm.pdf. Accessed October 20, 2017.

45. Gudka S. Pharmacy-based chlamydia screening [PhD's thesis]. Perth: University of Western Australia; 2014.

46. Bloomfield PJ, Kent C, Campbell D, Hanbrook L, Klausner JD. Community-based chlamydia and gonorrhea screening through the United States mail, San Francisco. Sex Transm Dis. 2002;29(5):294-297.

47. van Bergen JE, Postma MJ, Peerbooms PG, Spangenberg AC, Tjen-ATak J, Bindels PJ. Effectiveness and cost-effectiveness of a pharmacybased screening programme for Chlamydia trachomatis in a high-risk health centre population in Amsterdam using mailed home-collected urine samples. Int J STD AIDS. 2004;15(12):797-802.

48. Baraitser P, Pearce V, Holmes J, Horne N, Boynton P. Chlamydia testing in community pharmacies: evaluation of a feasibility pilot in south east London. Qual Saf Health Care. 2007;16(4):303-307.
49. Gudka S, Marshall L, Creagh A, Clifford RM. To develop and measure the effectiveness and acceptability of a pharmacy-based chlamydia screening intervention in Australia. BMJ Open. 2013;3(8):e003338.

50. Emmerton L, Skinner MB, Gardiner E, Nissen L, Debattista J. A trial of the distribution of chlamydia self-collection postal specimen kits from Australian community pharmacies. Sex Health. 2011;8(1):130-132.

51. Currie MJ, Deeks LS, Cooper GM, et al. Community pharmacy and cash reward: a winning combination for chlamydia screening? Sex Transm Infect. 2013;89(3):212-216.

52. Habel MA, Scheinmann R, Verdesoto E, Gaydos C, Bertisch M, Chiasson MA. Exploring pharmacy and home-based sexually transmissible infection testing. Sex Health. 2015;12(6):472-479.

53. Debattista J, Hayes M, Marshall P, Pouchkareff N, Gordon R, Priddle A. A trial of pharmacy-based testing for Chlamydia trachomatis using postal specimen kits. J Pharm Pract Res. 2017;47(1):41-46.

54. Taylor KL, Clifford RM, Marshall L. Acceptance of a chlamydia screening program in community pharmacies. $J$ Pharm Pract Res. 2007;37(4):287-291.

55. Thomas G, Humphris G, Ozakinci G, et al. A qualitative study of pharmacists' views on offering chlamydia screening to women requesting emergency hormonal contraception. BJOG. 2010;117(1):109-113.

56. Buchanan R, Hassan-Hicks P, Noble K, Grellier L, Parkes J, Khakoo SI. Integrating community pharmacy testing for hepatitis $\mathrm{C}$ with specialist care. Clin Pharmacist. 2016;8(8).

57. The Hepatitis C Trust. Pharmacy-based testing for hepatitis B and hepatitis C. Available from: http://www.hcvaction.org.uk/sites/default/files/ resources/Pharmacy-based\%20testing\%20for\%20hepatitis\%20B\%20 and $\% 20$ hepatitis $\% 20 \mathrm{C} \% 20 \% 28$ hep $\% 20 \mathrm{c} \% 20$ trust $\% 29$.pdf. Accessed October 30, 2017.

58. Calderon Y, Cowan E, Rhee JY, Brusalis C, Leider J. Counselor-based rapid HIV testing in community pharmacies. AIDS Patient Care STDs. 2013;27(8):467-473.

59. Weidle PJ, Lecher S, Botts LW, et al. HIV testing in community pharmacies and retail clinics: a model to expand access to screening for HIV infection. J Am Pharm Assoc (2003). 2014;54(5):486-492.

60. Gorostiza I, Elizondo López de Landache I, Braceras Izagirre L. Programa de cribado de VIH/sida en las oficinas de farmacia en la Comunidad Autónoma del País Vasco [HIV/AIDS screening program in community pharmacies in the Basque Country (Spain)]. Gac Sanit. 2013;27(2):164-166. Spanish.

61. Darin KM, Scarsi KK, Klepser DG, et al. Consumer interest in community pharmacy HIV screening services. J Am Pharm Assoc (2003). 2015;55(1):67-72.

62. Mayo Clinic [webpage on the Internet]. Gonorrhea - symptoms and causes. Available from: http://www.mayoclinic.org/diseases-conditions/gonorrhea/symptoms-causes/dxc-20258681. Accessed October 2, 2017.

63. Centers for Disease Control and Prevention [webpage on the Internet]. Drug-Resistant Gonorrhea - Antibiotic Resistance (AR) Solutions Initiative. Available from: https://www.cdc.gov/drugresistance/solutionsinitiative/drug-resistant-gonorrhea.html. Accessed October 2, 2017.

64. World Health Organization [webpage on the Internet]. WHO Guidelines for the treatment of Neisseria gonorrhoeae; 2016. Available from: http://www.who.int/reproductivehealth/publications/rtis/gonorrhoeatreatment-guidelines/en/. Accessed October 2, 2017.

65. Centers for Disease Control and Prevention [webpage on the Internet]. 2015 Sexually Transmitted Diseases Treatment Guidelines: Gonococcal Infections; 2015. Available from: https://www.cdc.gov/std/tg2015/ gonorrhea.htm. Accessed October 2, 2017.

66. Trecker MA, Dillon J-AR, Lloyd K, Hennink M, Waldner CL. Demographic and behavioural characteristics predict bacterial STI reinfection and coinfection among a cross-sectional sample of laboratory-confirmed gonorrhea cases in a local health region from Saskatchewan, Canada. Can J Public Health. 2015;106(2):17-21.

67. Wilson SP, Vohra T, Goldberg J, et al. Reliable rapid assay for gonorrhea and chlamydia in the emergency department. J Emerg Med. 2017;53(6):860-895. 
68. Lunny C, Taylor D, Hoang L, et al. Self-collected versus cliniciancollected sampling for chlamydia and gonorrhea screening: a systemic review and meta-analysis. PLoS One. 2015;10(7):e0132776.

69. Schachter J, McCormack WM, Chernesky MA, et al. Vaginal swabs are appropriate specimens for diagnosis of genital tract infection with Chlamydia trachomatis. J Clin Microbiol. 2003;41(8): 3784-3789.

70. World Health Organization [webpage on the Internet]. WHO guidelines for the treatment of Treponema pallidum (syphilis); 2016. Available from: http://www.who.int/reproductivehealth/publications/rtis/syphilistreatment-guidelines/en/. Accessed September 18, 2017.

71. Healthwise Staff, University of Michigan [webpage on the Internet]. Who is affected by syphilis. Available from: http://www.uofmhealth. org/health-library/hw195583. Accessed September 18, 2017.

72. Mayo Clinic [webpage on the Internet]. Syphilis - symptoms and causes. Available from: https://www.mayoclinic.org/diseases-conditions/syphilis/symptoms-causes/syc-20351756. Accessed September 18, 2017.

73. Lloyds Pharmacy [webpage on the Internet]. Tests for Sexually Transmitted Infections (STIs). Available from: http://www.lloydspharmacy. com/en/info/sti-tests. Accessed September 18, 2017.

74. AccuQuik Test Kit [webpage on the Internet]. Syphilis Test Kits. Available from: http://accuquiktestkits.com/our-test-kits/syphilis-test-kits. Accessed September 18, 2017.

75. BTNX Inc [webpage on the Internet]. Rapid Response(TM) Syphilis Antibody Cassette. Available from: https://www.btnx.com/ Product?id=1675. Accessed September 18, 2017.

76. World Health Organization [webpage on the Internet]. Hepatitis B fact sheet. Available from: http://www.who.int/mediacentre/factsheets/ fs204/en/. Accessed October 5, 2017.

77. Australian Family Physician [webpage on the Internet]. Hepatitis B serology. [Internet]; 2012. Available from: https://www.racgp.org.au/ afp/2012/april/hepatitis-b-serology/. Accessed October 5, 2017.
78. WebMD [webpage on the Internet]. Hepatitis B Guide: Treatment Overview. Available from: https://www.webmd.com/hepatitis/hepbguide/hepatitis-b-treatment-overview. Accessed October 6, 2017.

79. iCare [webpage on the Internet]. Hepatitis B Rapid Screen Test Instructions. Available from: https://www.TestKitMart.com. Accessed October 6, 2017

80. World Health Organization [webpage on the Internet]. Global health sector strategy on HIV, 2016-2021; 2016. Available from: http://apps.who. int/iris/bitstream/10665/246178/1/WHO-HIV-2016.05-eng.pdf?ua=1. Accessed October 28, 2017.

81. World Health Organization [webpage on the Internet]. HIV/AIDS. Available from: http://www.who.int/gho/hiv/en/. Accessed October 28, 2017.

82. World Health Organization [webpage on the Internet]. WHO: people most at risk of HIV are not getting the health services they need; 2014. Available from: http://www.who.int/mediacentre/news/releases/2014/ key-populations-to-hiv/en/. Accessed October 29, 2017.

83. Centers for Disease Control and Prevention [webpage on the Internet]. Sexually Transmitted Diseases (STDs); 2014. Available from: https:// www.cdc.gov/std/prevention/screeningreccs.htm. Accessed October 29, 2017.

84. BetterHealth channel [webpage on the Internet]. HIV and AIDS. Available from: https://www.betterhealth.vic.gov.au/health/conditionsandtreatments/hiv-and-aids. Accessed October 28, 2017.

85. Centers for Disease Control and Prevention [webpage on the Internet]. PrEP. Available from: https://www.cdc.gov/hiv/basics/prep.html. Accessed November 2, 2017.

86. Rothenberg RB, Scarlett M, del Rio C, Reznik D, O’Daniels C. Oral transmission of HIV. AIDS. 1998;12(16):2095-2105.

87. Centers for Disease Control and Prevention [webpage on the Internet]. Genital Herpes Screening FAQ. Available from: https://www.cdc.gov/ std/herpes/screening.htm. Accessed September 28, 2017.
Integrated Pharmacy Research and Practice

\section{Publish your work in this journal}

Integrated Pharmacy Research and Practice is an international, peer-reviewed, open access, online journal, publishing original research, reports, reviews and commentaries on all areas of academic and professional pharmacy practice. This journal aims to represent the academic output of pharmacists and pharmacy practice with particular focus on integrated care. All papers are carefully

\section{Dovepress}

peer reviewed to ensure the highest standards as well as ensuring that we are informing and stimulating pharmaceutical professionals. The manuscript management system is completely online and includes a very quick and fair peer-review system, which is all easy to use. Visit http://www.dovepress.com/ testimonials.php to read real quotes from published authors. 